\title{
Attentional biases in problem and non- problem gamblers
}

ARTICLE in JOURNAL OF AFFECTIVE DISORDERS · MARCH 2016

Impact Factor: 3.38 · DOI: 10.1016/j.jad.2016.03.009

READS

57

5 AUTHORS, INCLUDING:

Giovanna Nigro

Second University of Naples

35 PUBLICATIONS 159 CITATIONS

SEE PROFILE

\section{Marina Cosenza}

(4) Second University of Naples

17 PUBLICATIONS 33 CITATIONS

SEE PROFILE

\section{Mark D Griffiths}

Nottingham Trent University

754 PUBLICATIONS 15,054 CITATIONS

SEE PROFILE

Francesca D'Olimpio

Second University of Naples

32 PUBLICATIONS 613 CITATIONS

SEE PROFILE 


\title{
Attentional biases in problem and non-problem gamblers
}

\author{
Maria Ciccarelli ${ }^{1 *}$, Giovanna Nigro ${ }^{1}$, Mark D. Griffiths ${ }^{2}$, Marina Cosenza ${ }^{1}$, Francesca \\ D'Olimpio $^{1}$ \\ 1 Department of Psychology, Second University of Naples, Caserta, Italy \\ 2 Psychology Division, Nottingham Trent University, UK \\ * email address of the corresponding author: maria.ciccarelli@unina2.it
}

\begin{abstract}
Background: From a cognitive perspective, attentional biases are deemed as factors responsible in the onset and development of gambling disorder. However, knowledge relating to attentional processes in gambling is scarce and studies to date have reported contrasting results. Moreover, no study has ever examined which component and what type of bias are involved in attentional polarization in gambling. Methods: In the present study, 108 Italian participants, equally divided into problem and non-problem gamblers were administered a modified Posner Task, an attentional paradigm in which - through the manipulation of stimuli presentation time - it is possible to measure both initial orienting and maintenance of attention. In addition to the experimental task, participants completed self-report measures involving (i) craving (Gambling Craving Scale), (ii) depression, anxiety and stress (Depression Anxiety Stress Scale) and (iii) emotional dysregulation (Difficulties in Emotion Regulation Scale). Results: Analyses revealed facilitation in detecting gambling-related stimuli at the encoding level in problem gamblers but not in non-problem gamblers. Compared to non-problem gamblers, problem gamblers also reported higher levels of craving, emotional dysregulation, and negative mood states. Furthermore, all measures correlated with the gambling severity. Limitations: The use of indirect measure of attentional bias could be less accurate compared to direct measures. Conclusions: The facilitation in detecting gambling-related stimuli in problem gamblers and the correlation between subjective craving and facilitation bias suggests that attentional polarization could not be due to a conditioning process but that motivational factors such as craving could induce addicted-related seeking-behaviors.
\end{abstract}

Keywords: attentional bias; craving; emotions; gambling; problem gambling 


\section{HIGHLIGHTS}

Attentional bias, emotions and craving in gamblers and controls were assessed Problem gamblers showed facilitation bias compared to controls Problem gamblers reported high craving and negative mood states Problem gamblers showed high emotional dysregulation Correlations between facilitation bias and craving were observed 


\section{Introduction}

A large body of research has demonstrated that addictions are characterized by attentional biases toward addiction-related stimuli (see Field and Cox, 2008 for a review). Attentional bias can be defined as an automatic and uncontrollable tendency to allocate attention towards stimuli related to the individual's area of concern (Robinson and Berridge, 2008). Studies that have examined the attentional biases in addictions to nicotine (Ehrman et al., 2002), alcohol (Townshend and Duka, 2001), cannabis (Field et al., 2004), cocaine (Copersino et al., 2004), and gambling (Hønsi et al., 2013) have shown that addiction-related stimuli are processed more efficiently by addicted individuals, further strengthening irrational cognitions and maladaptive behaviors (Field and Cox, 2008; Field et al., 2009).

As proposed by Robinson and Berridge's $(1993,2008)$ incentive-sensitization model, stimuli associated with reward, through a classical conditioning process (such as substance-related or gambling-related stimuli), induce sensitization in the mesocorticolimbic dopamine system in the brain. This sensitization generates craving for addiction stimuli that captures attention and determines attentional biases, contributing to the maintenance of the disorder and leading to possible relapse. In the specific case of gambling, the continuous exposure to gambling can facilitate the detection of gambling-related stimuli in the environment, which can trigger a relapse through conditioned responses. In fact, once a gambling-related stimulus is detected, it can be processed automatically, making difficult to shift attention away from it. Moreover, since the attention is a limited resource, directing attention to a specific category of stimuli prevents the possibility of other stimuli being attended to (Kastner et al., 1998).

In the context of addictions that do not involve the ingestion of psychoactive substances, such as gambling disorder (American Psychiatric Association, 2013), knowledge relating to attentional processes is scarce. Studies carried out have reported contrasting results, probably due to the variety of instruments and/or to small sample sizes.

The most commonly used attentional instrument is the addiction Stroop Task (Cox et al., 2006). Using this instrument (in which people are required to read the color of the words, ignoring their semantic content), gamblers have been found to be slower to respond to gambling-related words and commit more errors compared to healthy controls (Boyer and Dickerson, 2003; McCusker and Gettings, 1997; Molde et al., 2010). Diskin and Hodgin's (1999) experimental study measured the reaction times of gamblers and non-gamblers to 
neutral stimuli such as a light during a gambling episode. They reported that regular gamblers took longer to react to irrelevant stimuli, demonstrating that gambling experience captures the attention of regular gamblers to a greater extent than among occasional gamblers.

Other research has found that problem gamblers display more signs of error-related attentional biases to gambling stimuli and commit more errors on response inhibition task in gambling-related trials (van Holst et al., 2012). However, some studies have failed to support the evidence of attentional biases in gambling. For example, Diskin and Hodgins (2001), in an effort to replicate their first (1999) experiment, confirmed only partially the pattern of results and (unlike their first study) did not find differences in the reaction times between occasional and problem gamblers. Similarly, Atkins and Sharpe (2003) examined reaction times with a modified Stroop Task in high- and low-frequency problem gamblers and found no difference between the two groups. Other studies have found no differences in the speed of reading relevant words between gamblers and control groups when the participants were not under the effect of dopamine agonist (Zack and Poulos, 2004) or antagonist (Zack and Poulos, 2007). These findings, that do not support the presence of attentional biases among gamblers, are probably due to the small samples (Atkins and Sharpe, 2003; Zack and Poulos, 2004,2007 ) and/or to the absence of problem gambling among participants (Atkins and Sharpe, 2003).

In addition, some issues remain unanswered. Firstly, it is not clear which attentional component is involved in gambling disorder. Researchers have distinguished between two components of selective attention: orienting and maintenance (Allport, 1989; LaBerge, 1995). Orienting refers to the rapid and automatic shift of attention (that occurs within $200 \mathrm{~ms}$ ), while maintenance refers to slow and continuous stimuli elaboration (that occurs within 500 ms or more) (Field and Cox, 2008; Field et al., 2009; Noel et al., 2006). To date, the majority of the studies evaluating attentional biases have employed the addiction Stroop Task that is unanimously considered a measure of the early stages of cognitive processing (Cox et al., 2006). Other studies (e.g., Grant and Bowling, 2015; Vizcaino et al., 2013) found no bias in attentional engagement and demonstrated that gambling frequency is associated with the maintenance of attention when using gambling-related stimuli. A recent study (Brevers et al., 2011b) used an attentional paradigm that allows the evaluation of these two attentional components. The study found that problem gamblers (compared to controls) were faster to detect gambling-related changes and showed biases in the initial orienting of attention. 
Furthermore, it is necessary to examine the nature of gambling-related attentional biases. Recently, three types of biases have been distinguished (Cisler and Koster, 2010): facilitation, that is the easiness to direct attention towards valenced stimuli (in this specific case, gambling-related cues in respect to neutral ones); avoidance, namely the tendency to avoid specific cues, allocating attention away from these; and disengagement, referring to a difficulty in diverting attention from these stimuli. Using a direct measure of attention (i.e., eye-tracking technology), Brevers and colleagues (2011a) observed a facilitation to respond to gambling-related pictures and a difficulty in disengaging attention away from these, namely a prolonged maintenance on gambling stimuli, with problem gamblers directing their gaze more frequently towards gambling stimuli.

Secondly, the relationship between craving and attentional biases is not clear. In Field's (2009) meta-analysis, a relationship between craving and attentional biases in drug addictions was detected, while, in the field of gambling, these results have not been confirmed (Brevers et al., 2011a; Wölfling et al., 2011) except in one study (i.e., Molde et al., 2010) that observed a correlation between attentional biases and abstinence. The relationship between craving and attentional biases on gambling-related stimuli needs clarifying as this could have important theoretical and practical implications. A correlation between these two constructs may indicate that attentional biases are not only the consequence of a classical conditioning process but that are associated with motivational states such as craving, and that a psychotherapeutic intervention on motivations to gamble may have an influence on biases.

Moreover, in the gambling studies literature, the role of gambling activity as a way to escape from negative emotions or mood is well known (Wood and Griffiths, 2007). In several studies that have examined gambling motivation, one of the most reported motivations is the use of gambling as a relief from negative psychological states (Blaszczynski and McConaghy, 1989; Blaszczynski and Nower, 2002; Dickerson et al., 1996). The refuge in gambling is not only a way to ameliorate mood states (e.g., Wood and Griffiths, 2007) but it may serve as a way to experience excitement and relieving boredom (Griffiths, 1995). Gambling involvement is also associated with the inability to manage emotions. For instance, Williams, Grisham, Erskine, and Cassedy (2012) reported that gamblers experience a high lack of emotional clarity and awareness and have a difficulty in adopting emotion-regulation strategies. Since the relationship between emotions and attentional bias has never been 
investigated, it is hypothesized that negative emotions and the inability to manage them in a healthy and functional way are likely to be associated with a tendency to allocate attention to very specific stimuli, such as gambling-related ones, and provide relief from them. Such issues have yet to be empirically addressed.

The comprehension of both the type of attentional biases and the attentional components involved in gambling disorder may help clinicians in the psychotherapeutic programs to aim their focus towards attentional modification. In light of this background, the purpose of the present study was threefold. Firstly, attentional biases in healthy controls and problem gamblers were measured, using a modified version of the Posner Task with exposure times assessing both early attentional processing and maintenance. Secondly the differentiation of three types of attentional biases was investigated. Finally, the relationships between these variables were analyzed.

Among indirect measures of biases, the choice to employ the Posner Task paradigm steams from two reasons. Firstly, through manipulation of cue presentation time, it allowed the assessment of biases at two levels: early orientation $(100 \mathrm{~ms})$ and maintenance of attention (500 ms). Secondly, the use of images (as opposed to words or other types of stimuli) is reported in the literature as being more suited to capturing attentional biases (Molde et al., 2010).

To the best of the authors' knowledge, the present study is the first to evaluate attentional processing, craving, emotional dysregulation, and emotional distress in both problem gamblers and healthy controls. It was hypothesized that there would be facilitation bias in problem gamblers but not in healthy controls. Finally, it was hypothesized that there would be positive correlations between attentional biases, craving, negative emotions, and emotional dysregulation.

\section{Method}

\section{Participants}

The sample comprised 108 male participants, with ages ranging from 21 to 63 years $(\mathrm{M}=36.80 ; \mathrm{SD}=11.52)$. The psychological literature has consistently reported that men are more likely than women to be problem gamblers (DSM-5; American Psychiatric Association, 2013). Participants were divided into two groups based on South Oaks Gambling Screen 
(SOGS; Lesieur and Blume, 1987). These were non-problem gamblers ( $\mathrm{N}=54)$ that scored from 0 to 2, gamblers at risk $(\mathrm{N}=19)$ with a score equal to $3-4$, and problem gamblers $(\mathrm{N}=35)$ that scored from 5 to 20 (the maximum being 17 in the present sample). Since no significant differences were found in attentional biases, emotional distress, emotional dysregulation, and craving between the 'gamblers at-risk' and the 'problem gamblers', they were combined into a single group ('problem gamblers') for the present study, in line with Lee et al.'s study (2011). Non-problem gamblers (NPGs; $\mathrm{N}=54$ ) and problem gamblers (PGs; N=54) groups were matched for age, (NPGs $=37.20$ years, $\mathrm{PGs}=36.39$ years; $\mathrm{t}_{106}=0.37, p=.71$ ) and number of years of education, (NPGs=11.57 years, PGs=11.72 years; $\mathrm{t}_{106}=-0.25, p=.80$ ).

\section{Measures}

Modified version of Posner Task (PT; Posner, 1980): The modified PT is a computerized version of a detecting attentional biases task. It was administered on a PC using the experimental software SuperLab 4.0 and the operating system Windows 8. The stimuli comprised 40 color pictures, chosen from non-copyrighted images on the Internet. Among these, 20 were 'gambling' pictures depicting slot machines, chips, lottery tickets, and 20 were 'neutral' pictures portraying petrol pumps, buttons and watches. The gambling and neutral pictures were matched for both color and shape. Each picture measured $350 \times 350$ pixels and a personal computer with a 15.6 " monitor was used to display the pictures on a grey background.

The task comprised 160 trials. The position of gambling images and the position of the dot were not balanced across trials so that $80 \%$ of the trials were valid (128 trials, 64 gambling and 64 neutral), where the target appears in the same location of the image that preceded it, and $20 \%$ of the trials were invalid ( 32 trials, 16 gambling and 16 neutral), where the target was presented in the opposite side (Posner, 1980). Each trial begins with a fixation point (“+”) (ITI; $1 \mathrm{~cm}$ in height) presented for $1000 \mathrm{~ms}$, followed by an image, in the left or right side of the screen for a fixed period of 100 or $500 \mathrm{~ms}$, after which it was immediately substituted by a dot (target). The dot is blue and appears in the same position of the picture (valid trial) or on the opposite side (invalid trial) for $1500 \mathrm{~ms}$ (see Figure 1).

Each image appears four times, as a valid and invalid trial, both for 100 and $500 \mathrm{~ms}$. The manipulation of the duration of the stimulus allows the investigation of different attentional 
components (e.g., Bradley et al., 2004; Field and Cox, 2008). A stimulus onset asynchrony (SOA) from 50 to $200 \mathrm{~ms}$ measures bias in the initial orienting of attention (facilitation and/or avoidance), because it allows only one shift of attention, whereas an SOA of $500 \mathrm{~ms}$ provides a longer time to make changes in gaze direction and reflects a bias in the maintenance or disengagement of attention (Field and Cox, 2008; Field et al., 2009).

Participants were seated $60 \mathrm{~cm}$ from the monitor and levelled with the center of the screen. They were asked to press a button on a keyboard (marked with white stickers) based on the location of the occurrence of the probe: "a" for left and "ù" for right. Participants were tested individually and instructed to respond to the probe as quickly and accurately as possible. Both accuracy and response times (RTs) were recorded.

Preliminary phase: validation of gambling-related pictures for the development of a modified version of Posner Task: As noted above, 80 images were chosen from the internet (40 gambling-related and 40 neutral pictures), and matched for color and shape. Twenty independent judges (10 males; mean age 26 years; $\mathrm{SD}=3.8$ ) were asked to evaluate the gambling relevance, pleasure, and arousal evoked by each image on a Likert scale from 0 ("not at all") to 9 ("very much"). For the construction of the modified version of PT, 20 gambling images were selected that received the highest scores on gambling relevance (mean gambling relevance $=7.26$ ), whereas the 20 images with the lowest scores was chosen as neutral (mean gambling relevance $=0.65$ ). Gambling and neutral images were no different on pleasure (gambling=2.26; neutral=2.66) and arousal (gambling $=2.55$; neutral=2.78).

South Oaks Gambling Screen (SOGS; Lesieur and Blume, 1987; Italian translation by Cosenza et al., 2014). The SOGS comprises 20 self-report items with dichotomous (yes/no) answers, based on the DSM criteria for pathological gambling (American Psychiatric Association, 1980) and assesses the frequency and the severity of gambling problems. The scores vary from 0 to 20 . Scores from 0 to 2 indicate no gambling problems, scores of 3-4 indicate the risk of developing gambling problems, and a score of 5 or above denotes problem and (probable) pathological gambling involvement. Typical items are "When you gamble, how often you go back another day to win back the money you lost?" and "Have people ever criticized your gambling?" SOGS items in the present study were found to have a high internal consistency reliability coefficient (Cronbach's alpha $=.92,95 \%$ CI [.91, .93]). 
Gambling Craving Scale (GACS; Young and Wohl, 2009; translated into Italian for the present study). The GACS is a multidimensional measure of gambling-related craving, comprising nine items assessing three different dimensions: anticipation (e.g., "If I had an opportunity of gamble right now, I would probably take it"), desire (e.g., "I need to gamble now"), and relief (e.g., "Gambling would make me less depressed"). For each item, respondents have to indicate agreement on a 7-point Likert scale from "strongly disagree" to "strongly agree". In the present study, internal consistency for the total scale $(\alpha=.89,95 \% \mathrm{CI}$ $[.85, .92])$ and for each scale was adequate: desire $(\alpha=.94,95 \%$ CI $[.92, .96])$, anticipation $(\alpha=.79,95 \%$ CI $[.72, .85])$, and relief $(\alpha=.77,95 \%$ CI $[.69, .84])$.

Difficulties in Emotion Regulation Scale (DERS; Gratz and Roemer, 2004; Italian validation by Sighinolfi et al., 2010). The DERS is a 36-item self-report questionnaire assessing several aspects of emotional dysregulation. It comprises six subscales: non-acceptance of emotional responses (e.g., “When I'm upset, I feel ashamed with myselffor feeling that way”), difficulties engaging in goal-directed behavior (e.g., "When I'm upset, I have difficulty focusing on other things"), Impulse control difficulties (e.g., "When I'm upset, I have difficulty controlling my behaviors"), lack of emotional awareness (e.g., "I care about what I am feeling", reverse coded), limited access to emotion regulation strategies (e.g., "When I'm upset, I believe that there is nothing I can do to make myself feel better") and lack of emotional clarity (e.g., "I have difficulty making sense out of my feelings"). Participants are asked to indicate how much each statement describes them with a 5-point Likert scale from 1 (almost never) to 5 (almost always). Higher scores suggest greater problems with emotion regulation. In the present study, Cronbach's alpha for the full scale was .91 (95\% CI [.89, $.93])$ and $.80,(95 \% \mathrm{CI}[.73, .85])$ for non-acceptance of emotional responses, .81 (95\% CI $[.75, .86])$ for difficulties engaging in goal-directed behavior, .83 (95\% CI $[.78, .88])$ for impulse control difficulties, .51 (95\% CI $[.36, .64])$ for lack of emotional awareness, .81 $(95 \%$ CI $[.76, .86])$ for limited access to emotion regulation strategies, and .71 (95\% CI [.61, .79]) for lack of emotional clarity.

Depression Anxiety Stress Scale (DASS-21; Henry and Crawford, 2005; Italian validation by Bottesi et al., 2015). The DASS-21 is the short and validated version of the original version (Lovibond and Lovibond, 1995) that contained 41 items. It assesses emotional feelings and mood states in the past two weeks with a 4-point scale ranging from 0 (did not apply to me at all) to 3 (applied to me very much, or most of the time). In the short version, there are 7 items 
for each of the three dimensions: depression (e.g., "I could see nothing to be hopeful about"), anxiety (e.g., "I felt scared without any good reason") and stress (e.g., "I tended to over-react to situations"). Higher scores indicate severe emotional distress. In this study, internal consistency for the total scale $(\alpha=.92,95 \%$ CI $[.90, .94])$ and for each subscale was adequate: depression $(\alpha=.86,95 \%$ CI $[.81, .89])$, anxiety ( $\alpha=.77,95 \%$ CI $[.70, .83])$, and stress $(\alpha=.86$, $95 \%$ CI $[.81, .89])$.

\section{Procedure}

The ethics committee of the research team's university department approved the present study, and informed consent was obtained prior to enrolment. Prior to testing, participants were assured that all measures were anonymous and that they could withdraw whenever they wanted. A code number was assigned to each participant to ensure anonymity. In a quiet room and individually, participants performed the Modified version of Posner Task and then filled in the questionnaires. After the experimental session, participants were debriefed and the purpose of the experiment was revealed and all their questions were answered. Participation in the study was voluntary and participants did not receive any reward.

\section{Data preparation}

Three types of attentional biases were calculated: facilitation, avoidance and disengagement, only for correct responses. Facilitation score was calculated from valid trials, subtracting reaction times for gambling-related stimuli from neutral stimuli. Positive facilitation scores indicate that attention is captured by gambling cues more greatly than by neutral images. Disengagement score was obtained by subtracting reaction times for neutral stimuli from gambling-related stimuli from invalid trials. Positive disengagement scores indicate disengagement biases (i.e. a prolonged maintenance of attention on gambling-related stimuli rather than neutral stimuli). Negative values of both facilitation and disengagement bias indicate avoidance biases, namely a tendency to avoid gambling stimuli. Values not different from zero are indicative of a lack of attentional biases, namely that there is no difference in the processing or in the maintenance of attention on gambling and non-gambling cues.

\section{Statistical analysis}

Data were analyzed with the IBM Statistical Package for the Social Sciences, version 20.0. The alpha significance level was set at .05 . A repeated analysis of variance $2 \times 2 \times 2 \times 2$ with Group (non-problem gamblers vs. problem gamblers) as between and Valence (gambling vs. 
neutral), Validity (valid vs. invalid), cue presentation Time (100 ms vs. $500 \mathrm{~ms}$ ) as within factors was conducted. A mixed ANOVA 2 x 2 was run on facilitation bias scores with one between-participant factor (Group: non-problem gamblers vs. problem gamblers) and two within-participant factors (facilitation bias at 100 and $500 \mathrm{~ms}$ ). The same mixed ANOVA was executed on Disengagement bias scores. Finally, a single-sample t-test was performed on facilitation and disengagement biases in order to assess if bias scores differed significantly from zero. Associations among measures were assessed with zero-order correlations.

\section{Results}

The percentage of incorrect trials for the entire sample was 3.36\%. Due to high percentage of missing data in the Posner Task, four participants (two belonging to the NPG group and two belonging to PG group) were excluded from the analysis on attentional bias scores. After selecting reaction times (RTs) only for correct responses, analyses on RTs were performed. A non-significant main effect of Valence $(\mathrm{F}(1,102)=0.10, p=.75)$, a main effect of Validity $\left(\mathrm{F}(1,102)=260.51, p<.001, \eta^{2}=.71\right)$, and a main effect of Time $\left(\mathrm{F}(1,102)=260.26, p<.001, \eta^{2}=.72\right)$ were found, with faster RTs for valid trials (RTs valid=389.34; RTs invalid=454.90) and for longer cue presentation $(\mathrm{RTs} 100 \mathrm{~ms}=443.74$; RTs $500 \mathrm{~ms}=400.51)$.

Valence $x$ Group $\left(\mathrm{F}(1,102)=0.55, p=.46, \eta^{2}=.00\right)$, Validity x Group $(\mathrm{F}(1,102)=0.41, p=.52)$, Time $x$ Group $(F(1,102)=0.19, p=.66)$, Valence $x$ Validity $(F(1,102)=0.90, p=.34)$, and Valence $\mathrm{x}$ Time $(\mathrm{F}(1,102)=1.99, p=.16)$ interactions were not significant. Validity $x$ Time interaction reached significance level $\left(\mathrm{F}(1,102)=6.88, p=.01, \eta^{2}=.06\right)$, indicating that $\mathrm{RTs}$ are faster for valid trials with longer presentation time (see Table 1).

The interactions Valence $\mathrm{x}$ Time $\mathrm{x}$ Group, $(\mathrm{F}(1,102)=0.33, p=.57)$, Validity $\mathrm{x}$ Time $\mathrm{x}$ Group, $(\mathrm{F}(1,102)=1.93, p=.17)$, Valence $\mathrm{x}$ Validity $\mathrm{x}$ Time, $(\mathrm{F}(1,102)=0.26, p=.61)$ and Valence $\mathrm{x}$ Validity $\mathrm{x}$ Time $\mathrm{x}$ Group, $(\mathrm{F}(1,102)=2.71, p=.10)$ fell short of the significance level. The ANOVA performed on facilitation bias scores showed a main effect of Time, $\left(F_{(1,102)}=4.04\right.$, $\left.p=.04, \eta^{2}=.04\right)$, a not significant main effect of Group, $\left(\mathrm{F}_{(1,102)}=0.01, p=.91\right)$ and a Group $\mathrm{x}$ Time interaction, $\left(\mathrm{F}(1,102)=5.14, p=.02, \eta^{2}=.05\right)$.

The same analysis was run on disengagement bias scores and revealed that neither the main effect of Time, $(\mathrm{F}(1,102)=0.28, p=.60)$, nor the main effect of Group, $\left(\mathrm{F}_{(1,102)}=0.96, p=.33\right)$, nor a Group $\mathrm{x}$ Time interaction, $\left(\mathrm{F}_{(1,102)}=0.37, p=.54\right)$ were significant. The single-sample t-test 
carried out on attentional bias scores revealed that NPGs showed neither facilitation bias (100 ms: $\left.\mathrm{t}_{51}=0.63, p=.53 ; 500 \mathrm{~ms}: \mathrm{t}_{51}=0.70, p=.48\right)$ nor disengagement bias (100 ms: $\mathrm{t}_{51}=-0.97$, $p=.34 ; 500$ ms: $\left.\mathrm{t}_{51}=0.24, p=.81\right)$. In the $\mathrm{PG}$ group only, a facilitation bias at $100 \mathrm{~ms}\left(\mathrm{t}_{51}=3.15\right.$, $p<.01)$, but not at $500 \mathrm{~ms}\left(\mathrm{t}_{51}=-0.97, p=.34\right)$, and no disengagement bias (100 ms: $\mathrm{t}_{51}=0.67$, $p=.51 ; 500$ ms: $\mathrm{t}_{51}=0.59, p=.56$ ) were observed (see Figure 2 ).

Positive correlations between facilitation bias at $100 \mathrm{~ms}$ and craving were observed. More specifically, facilitation bias at 100 ms correlated with GACS anticipation $(\mathrm{r}=.239 ; p=.01)$, GACS relief $(\mathrm{r}=.245 ; p=.01)$ and GACS total score $(\mathrm{r}=.263 ; p<.01)$. The association between facilitation bias at $100 \mathrm{~ms}$ and GACS desire $(\mathrm{r}=.171 ; p=.08)$ failed to reach significance. No other correlations were found.

\section{Discussion}

The aims of the present study were to identify which attentional component and what type of bias would be involved in the attentional bias in problem gamblers (PGs) compared to nonproblem gamblers (NPGs). To answer these questions, a modified Posner Task was used (Posner, 1980). Another issue concerned the relationship between attentional bias to gambling cues and craving, on which there is no consensus in literature. Other factors such as emotional competence and emotional distress were also examined.

The finding that PGs were faster in identifying gambling-related stimuli when presented at $100 \mathrm{~ms}$, with respect to neutral stimuli, indicated that attentional bias concerned the initial orientation of attention, namely the phase in which attention is captured by external stimuli, prior to any processing of it. This is in line with previous studies (Brevers et al., 2011a, 2011b; Field and Cox, 2008; McCusker and Gettings, 1997; Molde et al., 2010) that have reported a faster detection of gambling-related stimuli in problem gamblers but not in healthy control groups. Nevertheless, some past studies (i.e., Grant and Bowling, 2015; Vizcaino et al., 2013) observed biases towards gambling cues only in the maintenance of attention. However, it should be noted that the cue presentation time used in these studies (i.e., $500 \mathrm{~ms}$ or more) hampered the exploration of the early engagement of attention, allowing the attentional maintenance only. 
Identifying which type of bias (facilitation, avoidance, or disengagement) was present in gambling was the secondary aim of this study. The shorter reaction time in responding to gambling stimuli by problem gamblers indicated that they exhibited a facilitation bias, namely facilitation in perceiving gambling stimuli compared to other type of stimuli. This finding is consistent with Brevers et al.'s (2011a) study in which problem gamblers were found to be faster in detecting gambling-related changes compared to neutral changes. However, contrary to the present study, they observed an engagement of attention towards gambling stimuli: problem gamblers directed their gaze toward gambling more frequently and for a longer time. The difference in the pattern of results was probably due to the attentional bias paradigm. More specifically, Brevers and colleagues (2011a) used a direct measure (Eye Gaze Monitoring) that records eye first movements and fixations, whereas in the present study an indirect measure based on RTs was employed.

Correlational analysis revealed that facilitation in the initial orienting of attention was associated with motivational state of craving, contrary to previous studies that did not find an association between craving and attention (e.g. Brevers et al., 2011a; Wölfling et al., 2011). This was probably due to the sample characteristics. The Brevers and colleagues' study mainly comprised problem gamblers while, in the present study, $67 \%$ of the sample met the criteria for probable pathological gambling (SOGS $\geq 5$ ). As suggested by Young and Wohl (2009), GACS scale discriminates different levels of gambling severity, with craving scores being higher among those with a more severe gambling involvement.

The correlation between the subjective feeling of "wanting" gambling and attentional bias suggests that the readiness in identifying gambling stimuli is not only due to a conditioning process resulting from the continuous gambling experience, but also that the desire to gamble may facilitate the perception of gambling stimuli in the environment. Such findings can be explained by Robinson and Berridge's $(1993,2008)$ theory that views craving as important in determining addictive-related seeking-behaviors, in 'grabbing' addicts' attention and in fostering relapses. However, the hypothesis that emotional distress and dysregulation would be associated with a focus on gambling stimuli among PGs was not confirmed.

\section{Limitations}

Several limitations of the present study should be noted. The use of self-report measures represents a possible source of bias as social desirability, and the use of an indirect measure 
for the bias could be less accurate when compared to direct measures. An additional limitation of the current study is that there was no identification of a gamblers' preferred mode of gambling. A previous study has indicated that attentional biases are related not only to general gambling cues but also specific to gambling preference (McCusker and Gettings, 1997). Although the sample size in this study is greater than previous studies (Atkins and Sharp, 2003; Boyer and Dickerson, 2003; Brevers et al., 2011a; Diskin and Hodgins, 1999, 2001; McCusker and Gettings, 1997; Molde et al., 2010; Zack and Poulos, 2004, 2007), further research should include larger sample of gamblers, to ensure greater generalizability of the results. Further studies should also include a group of disordered gamblers with a DSM-5 diagnosis in order to understand attentional bias pattern in a sample varying from non-gamblers to pathological gamblers.

\section{Conclusion}

The present study adds knowledge about the time course of attentional biases in gambling. More specifically the study demonstrates that an attentional preference for gambling stimuli is present only in individuals who have developed a problematic involvement in gambling activities. The novel findings regarding the relationship between desire to gamble and preferential allocation on gambling stimuli suggest that psychotherapy should focus on motivational states in order to modify attentional biases that, if focused on gambling stimuli, could induce addiction-related seeking-behaviors and facilitate gambling. 


\section{References}

1. Allport, A., Visual attention. In M. I. Posner (Ed.), Foundations of cognitive science, 1989, MIT Press; Cambridge, MA, 631-682.

2. American Psychiatric Association, Diagnostic and Statistical Manual of Mental Disorders, Third edition, 1980, APA; Washington DC.

3. American Psychiatric Association, Diagnostic and Statistical Manual of Mental Disorders, Fifth edition, 2013, APA; Washington DC.

4. Atkins, G., Sharpe, L., Cognitive biases in problem gambling. Gambl. Res.: J. Natl. Assoc. Gambl. Stud., 15(2), 2003, 35-43.

5. Blaszczynski, A., McConaghy, N., Anxiety and/or depression in the pathogenesis of addictive gambling. Int. J. Addict., 24(4), 1989, 337-350.

6. Blaszczynski, A., Nower, L., A pathways model of problem and pathological gambling. Addiction, 97(5), 2002, 487-499.

7. Bottesi, G., Ghisi, M., Altoè, G., Conforti, E., Melli, G., Sica, C., The Italian version of the Depression Anxiety Stress Scales-21: factor structure and psychometric properties on community and clinical samples. Compr. Psychiatry, 60, 2015, 170-181.

8. Boyer, M., Dickerson, M., Attentional bias and addictive behaviour: automaticity in a gambling-specific modified stroop task. Addiction, 98(1), 2003, 61-70.

9. Bradley, B., Field, M., Mogg, K., De Houwer, J., Attentional and evaluative biases for smoking cues in nicotine dependence: component processes of biases in visual orienting. Behav. Pharm., 15(1), 2004, 29-36.

10. Brevers, D., Cleeremans, A., Bechara, A., Laloyaux, C., Kornreich, C., Verbanck, P., Noël, X., Time course of attentional bias for gambling information in problem gambling. Psychol. Addict. Behav., 25(4), 2011a, 675-682.

11. Brevers, D., Cleeremans, A., Tibboel, H., Bechara, A., Kornreich, C., Verbanck, P., Noël, $\mathrm{X}$., Reduced attentional blink for gambling-related stimuli in problem gamblers. $J$. Behav. Ther. Exp. Psychiatry, 42(3), 2011b, 265-269.

12. Cisler, J.M., Koster, E.H., Mechanisms of attentional biases towards threat in anxiety disorders: an integrative review. Clin. Psychol. Rev., 30(2), 2010, 203-216.

13. Copersino, M.L., Serper, M.R., Vadhan, N., Goldberg, B.R., Richarme, D., Chou, J.C.Y., Stitzer M., Cancro, R., Cocaine craving and attentional bias in cocaine-dependent schizophrenic patients. Psychiatry Res., 128(3), 2004, 209-218.

14. Cosenza, M., Matarazzo, O., Baldassarre, I., Nigro, G., 2014. Deciding with (or without) the Future in Mind: Individual Differences in Decision-Making. In Recent Advances of Neural Network Models and Applications (pp. 435-443). Springer International Publishing.

15. Cox, W.M., Fadardi, J.S., Pothos, E.M., The addiction-stroop test: theoretical considerations and procedural recommendations. Psychol. Bull., 132(3), 2006, 443-476.

16. Dickerson, M.G., Baron, E., Hong, S.M., Cottrell, D., Estimating the extent and degree of gambling related problems in the Australian population: a national survey. J. Gambl. Stud., 12(2), 1996, 161-178.

17. Diskin, K.M., Hodgins, D.C., Narrowing of attention and dissociation in pathological video lottery gamblers. J. Gambl. Stud., 15(1), 1999, 17-28.

18. Diskin, K.M., Hodgins, D.C., Narrowed focus and dissociative experiences in a community sample of experienced video lottery gamblers. Can. J. Behav. Sci., 33(1), 2001, 58-64.

19. Ehrman, R.N., Robbins, S.J., Bromwell, M.A., Lankford, M.E., Monterosso, J.R., O'Brien, C.P., Comparing attentional bias to smoking cues in current smokers, former 
smokers, and non-smokers using a dot-probe task. Drug Alcohol Depend., 67(2), 2002, 185-191.

20. Field, M., Cox, W.M., Attentional bias in addictive behaviors: a review of its development, causes, and consequences. Drug Alcohol Depend., 97(1), 2008, 1-20.

21. Field, M., Mogg, K., Bradley, B.P., Cognitive bias and drug craving in recreational cannabis users. Drug Alcohol Depend., 74(1), 2004, 105-111.

22. Field, M., Munafò, M.R., Franken, I.H., A meta-analytic investigation of the relationship between attentional bias and subjective craving in substance abuse. Psychol. Bull., 135(4), 2009, 589-607.

23. Grant, L. D., Bowling, A. C., Gambling attitudes and beliefs predict attentional bias in non-problem gamblers. J. Gambl. Stud., 31(4), 2015, 1487-1503.

24. Gratz, K.L., Roemer, L., Multidimensional assessment of emotion regulation and dysregulation: development, factor structure, and initial validation of the difficulties in emotion regulation scale. J. Psychopathol. Behav. Assess., 26(1), 2004, 41-54.

25. Griffiths, M., The role of subjective mood states in the maintenance of fruit machine gambling behavior. J. Gambl. Stud., 11(2), 1995, 123-135.

26. Henry, J.D., Crawford, J.R., The short-form version of the Depression Anxiety Stress Scales (DASS-21): construct validity and normative data in a large non-clinical sample. Br. J. Clin. Psychol., 44(2), 2005, 227-239.

27. Hønsi, A., Mentzoni, R. A., Molde, H., Pallesen, S., Attentional bias in problem gambling: A systematic review. J. Gambl. Stud., 29(3), 2013, 359-375.

28. Kastner, S., De Weerd, P., Desimone, R., Ungerleider, L. G., Mechanisms of directed attention in the human extrastriate cortex as revealed by functional MRI. Science, 282, 1998, 108-111.

29. LaBerge, D., Attentional Processing: The brain's art of Mindfulness 2, 1995, Harvard University Press; Harvard, MA.

30. Lee, G. P., Storr, C. L., Ialongo, N. S., Martins, S. S., Compounded effect of early adolescence depressive symptoms and impulsivity on late adolescence gambling: a longitudinal study. J. Adolesc. Health, 48(2), 2011, 164-169.

31. Lesieur, H.R., Blume, S.B., The South Oaks Gambling Screen (SOGS): a new instrument for the identification of pathological gamblers. Am. J. Psychiatry, 144(9), 1987, 11841188.

32. Lovibond, P. F., Lovibond, S. H., The structure of negative emotional states: comparison of the Depression Anxiety Stress Scales (DASS) with the Beck depression and anxiety inventories. Behav. Res. Ther., 33(3), 1995, 335-343.

33. McCusker, C.G., Gettings, B., Automaticity of cognitive biases in addictive behaviors: further evidence with gamblers. Br. J. Clin. Psychol., 36(4), 1997, 543-554.

34. Molde, H., Pallesen, S., Sætrevik, B., Hammerborg, D.K., Laberg, J.C., Johnsen, B.H., Attentional biases among pathological gamblers. Int. Gambl. Stud., 10(1), 2010, 45-59.

35. Noël, X., Colmant, M., Van Der Linden, M., Bechara, A., Bullens, Q., Hanak, C., Verbanck, P., Time course of attention for alcohol cues in abstinent alcoholic patients: the role of initial orienting. Alcoholism: Clin. Exp. Res., 30(11), 2006, 1871-1877.

36. Posner, M.I., Orienting of attention. Q J. Exp. Psychol., 32(1), 1980, 3-25.

37. Robinson, T.E., Berridge, K.C., The neural basis of drug craving: an incentivesensitization theory of addiction. Brain Res. Rev., 18(3), 1993, 247-291.

38. Robinson, T.E., Berridge, K.C., Review. The incentive sensitization theory of addiction: some current issues. Philos. Trans. R. Soc. Lond. B. Biol. Sci., 363(1507), 2008, 31373146. 
39. Sighinolfi, C., Norcini Pala, A., Chiri, L.R., Marchetti, I., Sica, C., Difficulties in emotion regulation scale (DERS): the Italian translation and adaptation. Psicoter Cogn. Comportamentale, 16(2), 2010, 141-170.

40. Townshend, J., Duka, T., Attentional bias associated with alcohol cues: differences between heavy and occasional social drinkers. Psychopharmacology, 157(1), 2001, 6774.

41. van Holst, R. J., Lemmens, J. S., Valkenburg, P. M., Peter, J., Veltman, D. J., Goudriaan, A. E., Attentional bias and disinhibition toward gaming cues are related to problem gaming in male adolescents. J. Adolesc. Health, 50(6), 2012, 541-546.

42. Vizcaino, E.J.V., Fernandez-Navarro, P., Blanco, C., Ponce, G., Navio, M., Moratti, S., Rubio, G., Maintenance of attention and pathological gambling. Psychol. Addict. Behav., 27(3), 2013, 861.

43. Williams, A.D., Grisham, J.R., Erskine, A., Cassedy, E., Deficits in emotion regulation associated with pathological gambling. Br. J. Clin. Psychol., 51(2), 2012, 223-238.

44. Wölfling, K., Mörsen, C.P., Duven, E., Albrecht, U., Grüsser, S.M., Flor, H., To gamble or not to gamble: at risk for craving and relapse-learned motivated attention in pathological gambling. Biol. Psychol., 87(2), 2011, 275-281.

45. Wood, R.T., Griffiths, M.D., A qualitative investigation of problem gambling as an escape-based coping strategy. Psychol. Psychother., 80(1), 2007, 107-125.

46. Young, M.M., Wohl, M.J., The Gambling Craving Scale: psychometric validation and behavioral outcomes. Psychol. Addict. Behav., 23(3), 2009, 512-522.

47. Zack, M., Poulos, C.X., Amphetamine primes motivation to gamble and gamblingrelated semantic networks in problem gamblers. Neuropsychopharmacology, 29(1), 2004, 195-207.

48. Zack, M., Poulos, C.X., A D2 antagonist enhances the rewarding and priming effects of a gambling episode in pathological gamblers. Neuropsychopharmacology, 32(8), 2007, 1678-1686. 
Table 1. Mean and SD in the modified Posner Task as a function of gambling group, validity, valence and cue presentation time.

\begin{tabular}{lllcc} 
& & & NPG $(\mathbf{N}=\mathbf{5 4})$ & PG $(\mathbf{N}=\mathbf{5 4})$ \\
\cline { 3 - 5 } & & M (SD) & M (SD) \\
\hline $\mathbf{1 0 0} \mathbf{~ m s}$ & Valid & Gambling & $405.44(58.77)$ & $416.88(71.20)$ \\
& & Neutral & $407.17(54.17)$ & $427.35(68.04)$ \\
& \multirow{2}{*}{ Invalid } & Gambling & $456.38(58.46)$ & $489.63(73.72)$ \\
& & Neutral & $462.19(64.17)$ & $484.83(70.00)$ \\
\multirow{2}{*}{} & \multirow{2}{*}{ Valid } & Gambling & $350.19(59.32)$ & $379.81(76.11)$ \\
& \multirow{2}{*}{ Invalid } & Neutral & $352.94(58.62)$ & $374.96(60.77)$ \\
& & Gambling & $425.19(43.50)$ & $450.79(64.28)$ \\
& & Neutral & $423.75(48.34)$ & $446.46(58.72)$ \\
\hline
\end{tabular}


VALID TRIAL

INVALID TRIAL

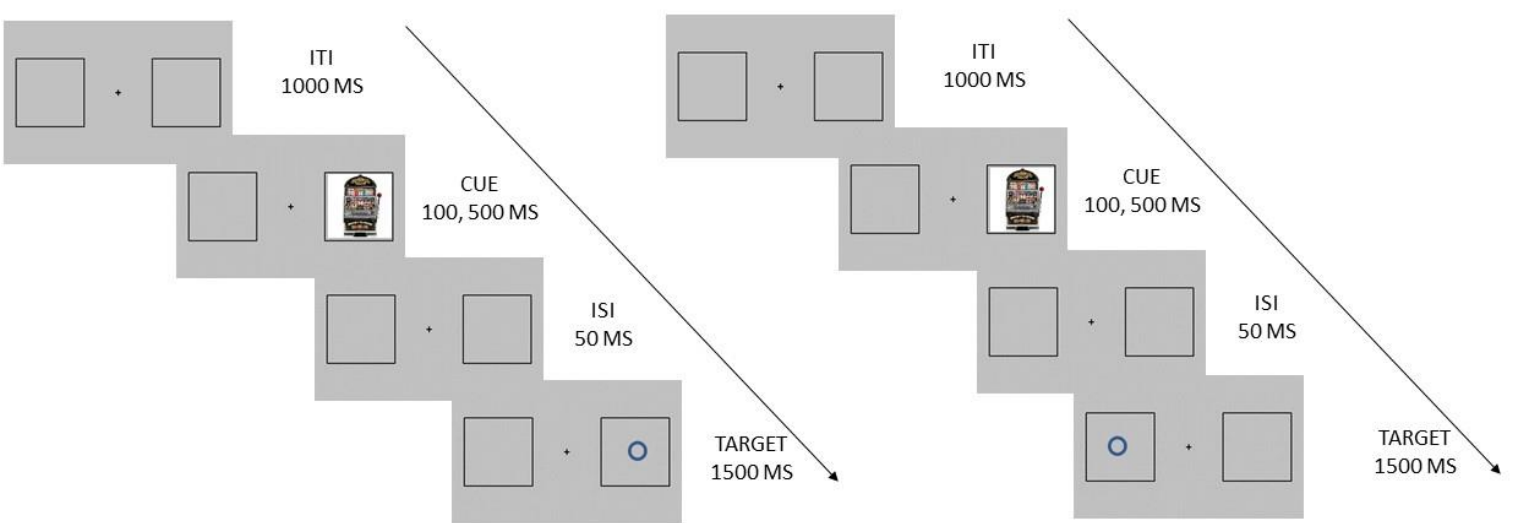

Figure 1. Modified version of Posner Task screen. The two columns illustrate a valid and an invalid trial, respectively. 


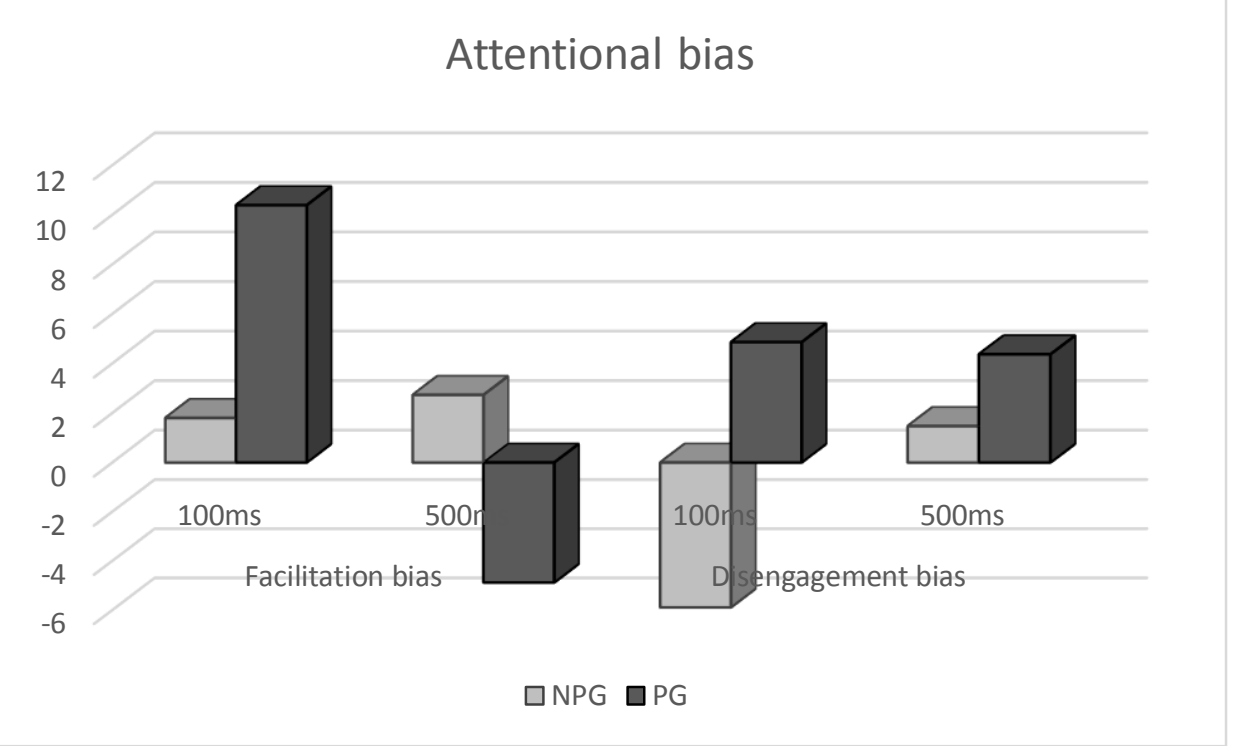

Figure 2. Attentional bias scores as a function of Group (NPG=non-problem gamblers, $\mathrm{PG}=$ problem gamblers). 